\title{
An Outbreak of Histomoniasis in Backyard Sanhuang Chickens
}

\author{
Dandan Liu' ${ }^{1,2}$, Lingming Kong ${ }^{1,2}$, Jianping Tao ${ }^{1,2}$, Jinjun $\mathrm{Xu}^{1,2, *}$ \\ 'Jiangsu Co-innovation Center for Prevention and Control of Important Animal Infectious Diseases and Zoonoses, Yangzhou, Jiangsu Province 225009, \\ P. R. China; 'Ministry of Education Key Lab for Avian Preventive Medicine, Yangzhou University, Yangzhou, Jiangsu Province 225009, P. R. China
}

\begin{abstract}
Histomonas meleagridis is a facultative anaerobic parasite, which can cause a common poultry disease known as histomoniasis. The species and age of the birds impacts on the susceptibility, with turkey being the most susceptible species. Chickens are less susceptible to $H$. meleagridis than turkeys and usually serve as reservoir hosts. Here, the diagnosis of an outbreak of histomoniasis in backyard Sanhuang chickens is described. The primary diagnosis was made based on clinical symptoms, general changes at necropsy, histopathology, and the isolation and cultivation of parasites. The pathogen was further confirmed by cloning, PCR identification, and animal inoculation tests. A strain of $H$. meleagridis, named HM-JSYZ-C, with a higher pathogenicity level in chickens was obtained. The study lays a foundation for further investigations into $H$. meleagridis and histomoniasis in chickens.
\end{abstract}

Key words: Histomonas meleagridis, Sanhuang, chicken, PCR, histopathology

\section{INTRODUCTION}

Histomoniasis, also known as "blackhead disease" or "enterohepatitis", is a parasitic disease in gallinaceous birds caused by Histomonas meleagridis [1]. The main symptoms of histomoniasis include liver necrosis, cecal enlargement and sulfur-yellow stools [1]. For food safety considerations, most of the chemicals used to treat the disease in Europe and the USA have been banned or restricted, resulting in serious epidemics in turkey flocks in recent years [2].

There have been reports on the prevalence and incidence of this disease in chicken flocks worldwide in recent years. It is generally believed that chickens are the reservoir hosts for $H$. meleagridis because they are less susceptible than turkeys and their mortality rate after infection is usually lower than $10 \%-$ far below the $100 \%$ in turkeys $[3,4]$. Therefore, histomoniasis in chicken flocks is often ignored by farm owners and veterinarians. In recent years, the chicken industry in China has developed rapidly, having both the greatest number and variety of chickens worldwide. In the past, there was almost no inci-

\footnotetext{
- Received 18 April 2018, revised 12 October 2018, accepted 14 October 2018.

*Corresponding author (jjxu@yzu.edu.cn)

(C) 2018, Korean Society for Parasitology and Tropical Medicine

This is an Open Access article distributed under the terms of the Creative Commons Attribution Non-Commercial License (http://creativecommons.org/licenses/by-nc/4.0) which permits unrestricted non-commercial use, distribution, and reproduction in any medium, provided the original work is properly cited.
}

dence of histomoniasis owing to the wide use of chemicals and drugs. With the restricted use of drugs in feed and the promotion of ecological farming methods, such as free ranging in recent years, outbreaks and epidemics of the disease in chicken flocks have constantly occurred in China [5].

Sanhuang (SH) chicken is a Chinese indigenous breed which has uniform body fat, high intramuscular fat and is a popular food product. Currently, large numbers of this popular poultry breed are raised in China, and it is mostly free ranging [6]. Here, we report an outbreak of histomoniasis with a high mortality in a SH chicken flock free ranging in the backyard of a farmer. The pathogen of histomoniasis was identified by histopathology, isolation and in vitro culturing, PCR, and animal inoculation tests.

\section{CASE RECORD}

\section{Clinical symptoms}

A farmer in Hanjiang District, Yangzhou City, Jiangsu Province, raised more than 500 60-day-old SH chickens. The chickens were free ranged in the backyard during the day and fed with rice and grass (Fig. 1A), and they entered the hen house freely at night. On 12 July 2015, a disease began to occur in the chicken flock, with symptoms that included crouched heads, drooping wings and the excretion of watery yellowgreen diarrhea (Fig. 1B). In total, 15-20 chickens died daily. 

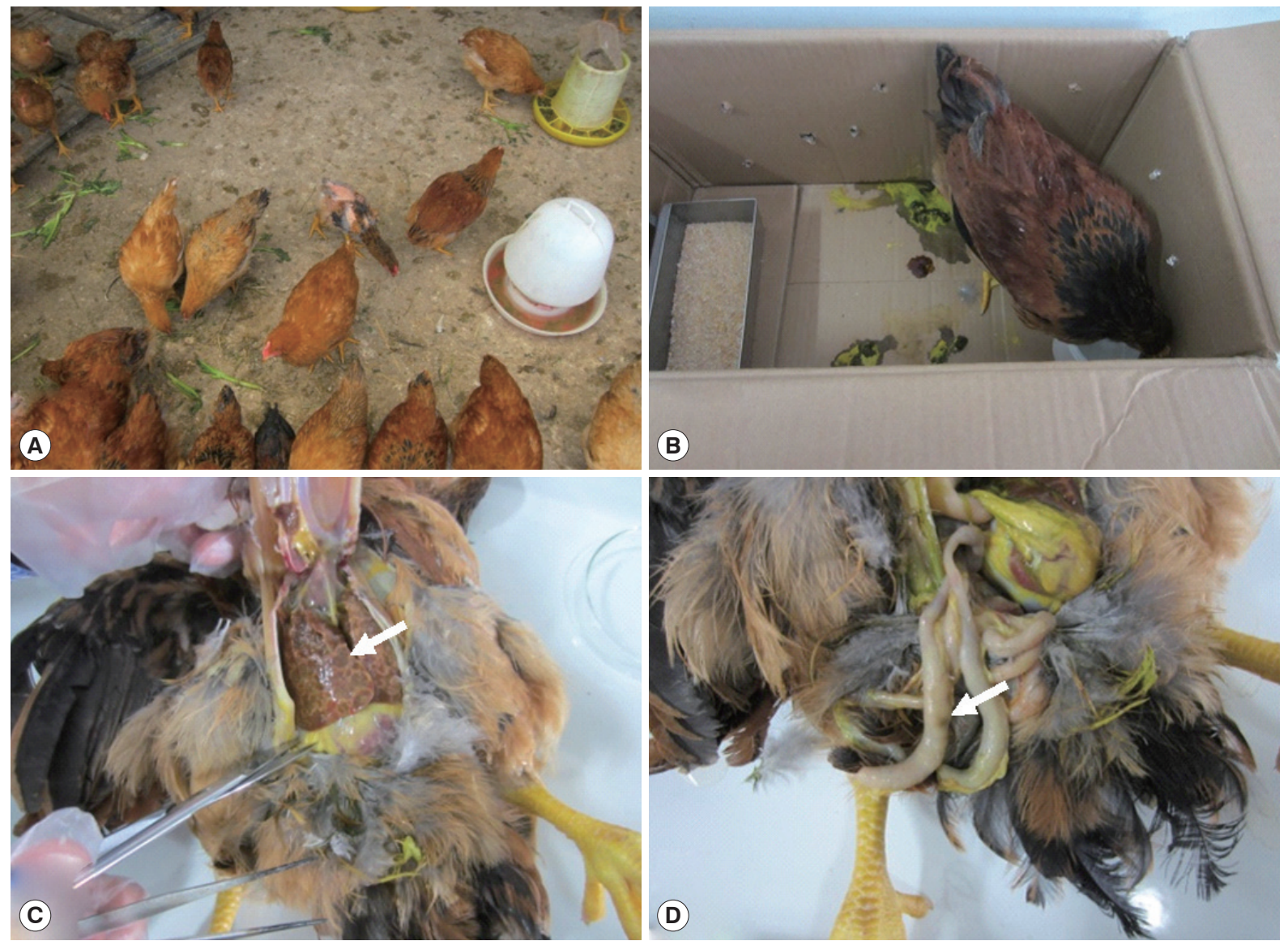

Fig. 1. Clinical syndromes and post-mortem findings of Sanhuang chickens. (A) Free range system. (B) Depression and yellow-green feces of chickens. (C) Severe necrosis appeared as sulfur-colored rings in the liver (white arrow). (D) Swelling and caseous cores in the ceca (white arrow).

On 22 July 2015, after more than 200 chickens had already died, the owner visited the Animal Disease Diagnosis Center of the College of Veterinary Medicine, Yangzhou University, and there were still nearly 30 sick chickens. After a consultation and field investigation, the flock was vaccinated against infectious diseases, such as H5-subtype bird flu, Newcastle disease, infectious bronchitis and bursal disease, in accordance with local immunization procedures. Albendazole (Tongren Pharmaceutical Co. Ltd., Shanghai, China) was orally administered once to 45-day-old chickens at a dose of $20 \mathrm{mg} / \mathrm{kg}$ of body weight to expel parasites, and diclazuril (Baoling Animal Health Product Co. Ltd., Tianjin, China) was added to the drinking water at a dose of $0.5 \mathrm{ppm}$ per chicken to prevent coccidiosis. Doxycycline (Tongren Pharmaceutical Co. Ltd., Shanghai, China) was used to treat the remaining sick chickens at a dose of $25 \mathrm{mg} / \mathrm{kg}$ of body weight, twice daily for 5 days, with no obvious effects.

\section{Pathological changes at necropsy}

Four dead chickens were dissected, and the pathological changes were similar. The livers were enlarged and ulcerated lesions with circular depressions had formed on the surface. The lesions were light yellow or yellowish green in the center, the peripheral edges were uplifted, and they had a unique crater-like shape (Fig. 1C). The ceca were swollen (Fig. 1D) and contained casein-like emboli. After the removal of the contents, necrosis and ulcers of the ceca were seen. No significant changes were found in other organs. No trematode, cestode, nematode or coccidial oocysts were found in the contents of the small intestines and ceca by conventional parasitic examinations.

\section{Histopathologic examination}

Appropriate amounts of liver and cecal tissues were cut and observed in the hematoxylin and eosin stained tissue sections. 

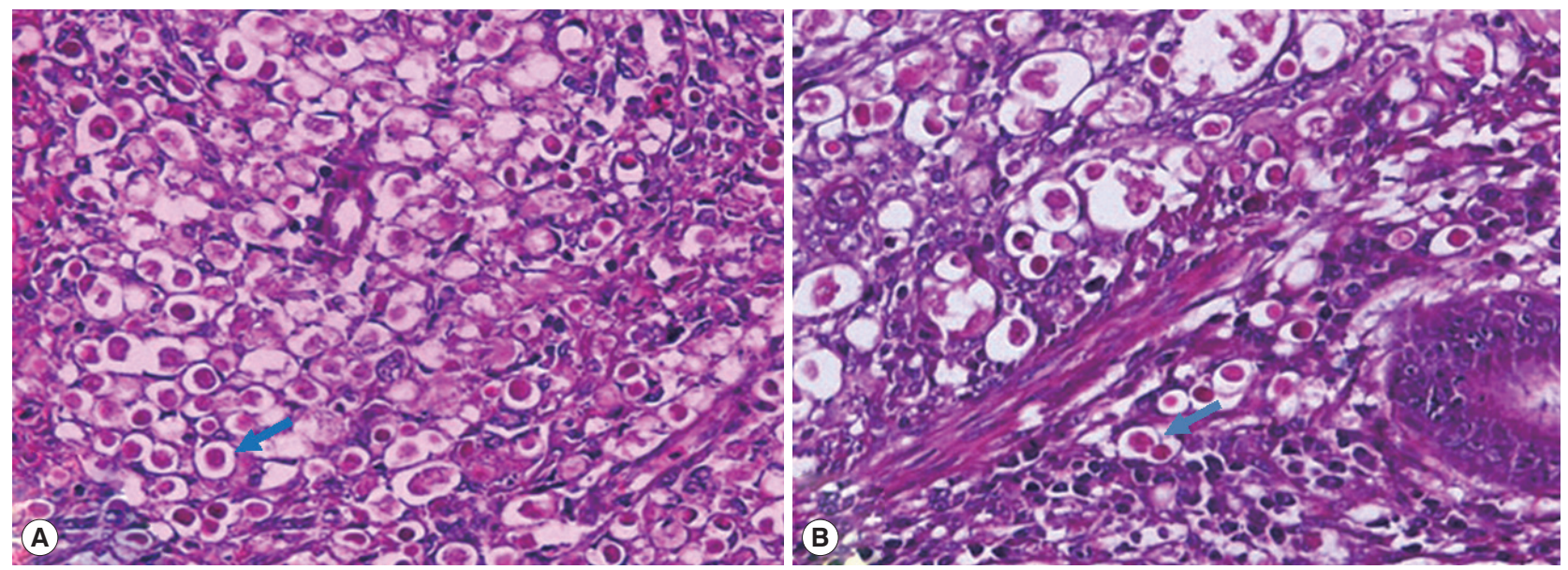

Fig. 2. Histopathological changes in livers and ceca from the dead Sanhuang chickens. (A) Numerous histomonads (blue arrow) in the liver (HE, ×400). (B) Numerous histomonads (blue arrow) in ceca (HE, × 400).

Then, the sections were mounted with neutral balsam for microscopic examination. A large number of circular eosinophilic histomonad-like parasites appeared in the liver and cecal tissues (Fig. 2).

\section{Isolation and identification of parasites}

Approximately $1 \mathrm{~g}$ of necrotic tissues from diseased chicken livers were aseptically removed and placed into a sterile $1.5 \mathrm{ml}$ Eppendorf tubes. The tissues were smashed and inoculated into $10 \mathrm{ml}$ medium that was preheated to $40^{\circ} \mathrm{C}$, and then incubated anaerobically in an incubator at $40^{\circ} \mathrm{C}$. The medium consisted of $9 \mathrm{ml}$ of M199 medium (Gibco, Grand Island, New York, USA), $1 \mathrm{ml}$ of horse serum (Gibco), and $11 \mathrm{mg}$ of rice flour (Sigma, St. Louis, Missouri, USA), and was inoculated with a ring of cecal bacteria grown on a Columbia blood agar plate. The cecal bacteria were obtained by inoculating Colombia blood agar plate with the cecal contents of healthy chickens and storing them overnight at $37^{\circ} \mathrm{C}$. A microscopic examination after $36 \mathrm{hr}$ revealed that the isolated parasites were round or oval. There were rice flour particles in the cells that were refractive, $10-20 \mu \mathrm{m}$ in size, with a pendulum-like movement that was slow and weak (Fig. 3). Primers were designed targeting the H. meleagridis $18 \mathrm{~S}$ rRNA gene (P1: 5' -GAAAGCATCTATCAAGTGGAA-3' and P2: 5' -GATCTTTTCAA ATTAGCTTTAAA-3') [7]. The primers were synthesized by Sangon Biotech (Shanghai, China). The parasite DNA was extracted using an EasyPure Genomic DNA Kit (TransGen Biotec, Beijing, China) and was identified by PCR. The sequencing of the 3 PCR products (each performed 3 times) showed 100\%

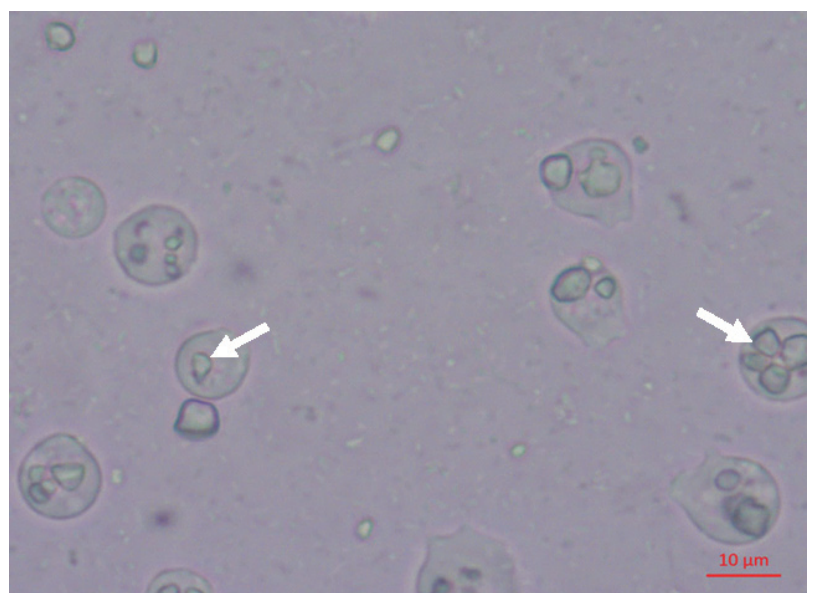

Fig. 3. Light micrograph of cloned Histomonas meleagridis cultures; parasites showed the phagocytosis of rice starch granules (white arrows).

identity levels and that a specific fragment of $576 \mathrm{bp}$ was amplified, named H. meleagridis SH (GenBank Accession No. MH449665). The $18 \mathrm{~S}$ rRNA sequences were added to an existing database of 10 other parabasalid sequences, including strains isolated from different hosts (turkey and chicken) and regions (France, Australia and America), as well as 2 outgroup sequences. Accession numbers for the sequences used in the phylogenetic analyses are indicated in Fig. 4. A maximum likelihood analysis was carried out with MEGA. The sequence of H. meleagridis SH was $100 \%$ homologous to the $18 \mathrm{~S}$ rRNA sequence of H. meleagridis (JX963670) in GenBank. Combining the morphological observations and phylogenetic analysis, the isolated parasites were identified as $H$. meleagridis. 


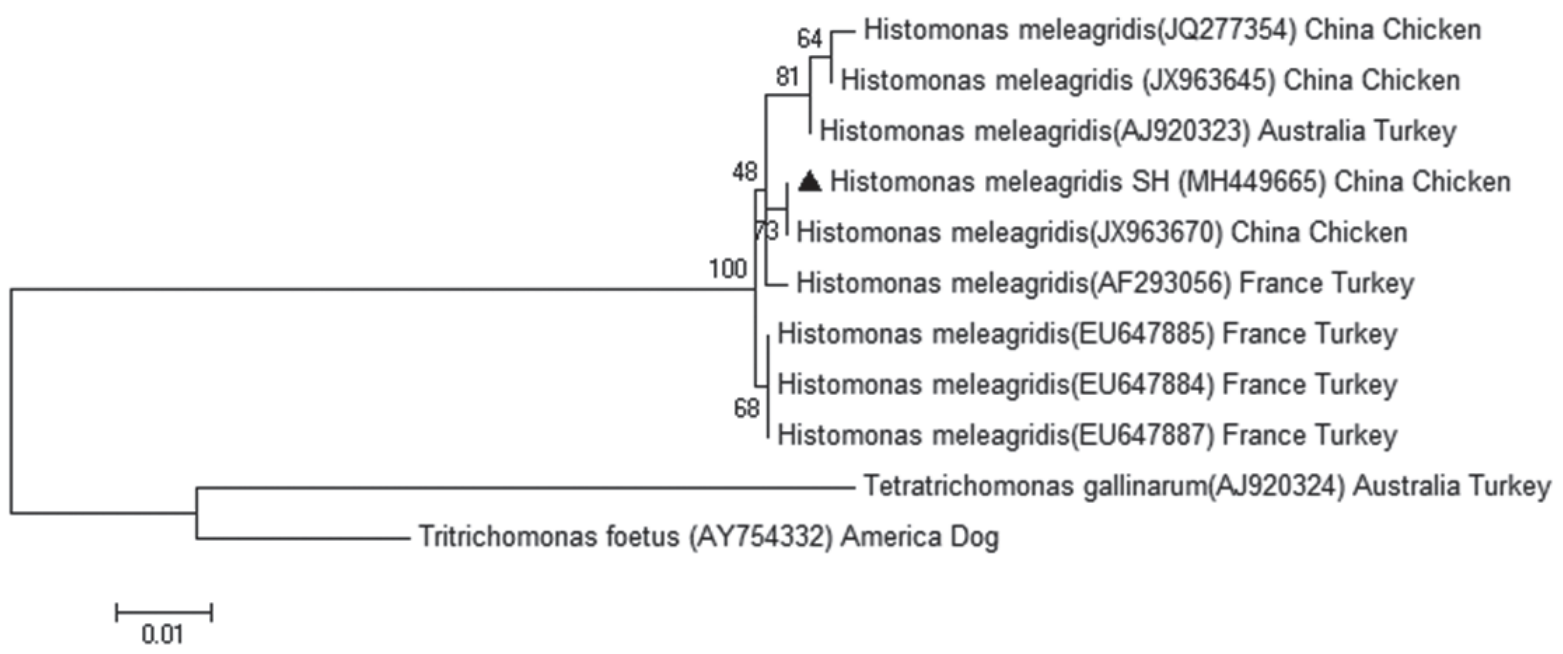

Fig. 4. Maximum likelihood tree of parabasalids based on 18S rRNA gene sequences. A Histomonas meleagridis SH sequence obtained in this study is indicated by a black triangle. Number on a individual node indicates a bootstrap value. Nodes with values below 50\% are not shown. Scale bar indicates 0.01 substitutions (corrected) per base pair.
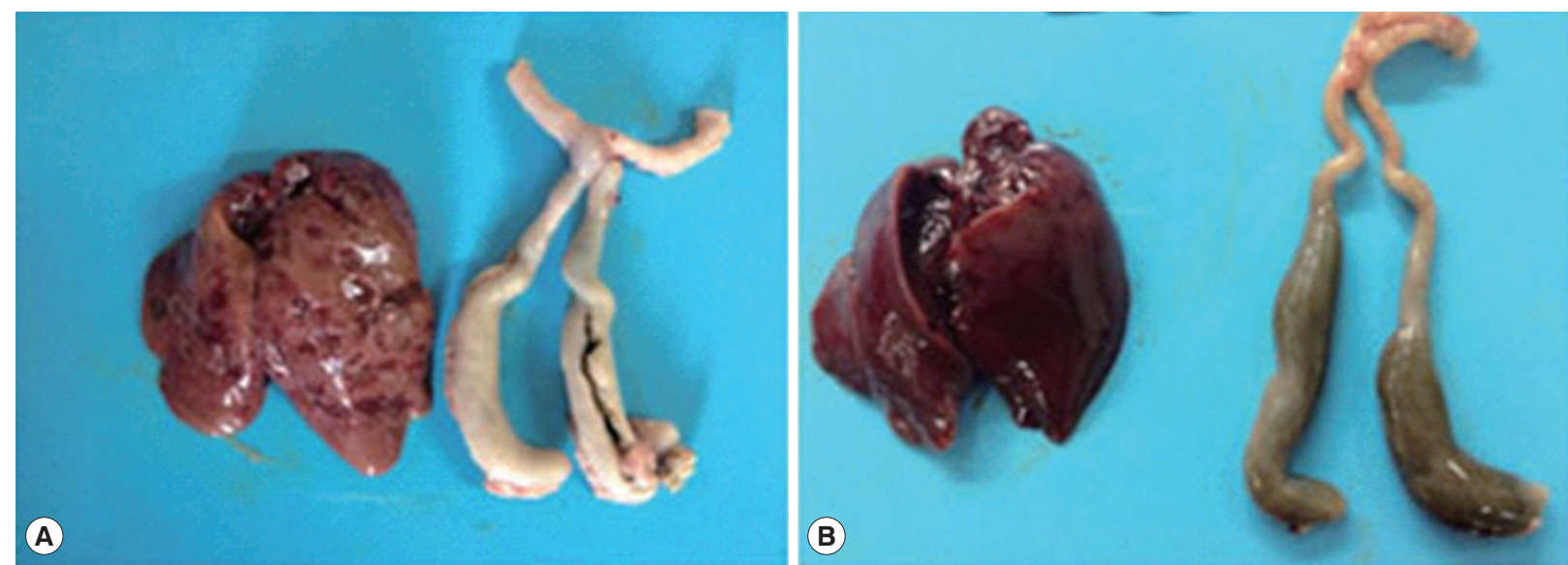

Fig. 5. Results of the animal inoculation test. (A) Liver and ceca of a chicken intra-cloacally infected with isolated Histomonas meleagridis. Typical macroscopic changes associated with histomoniasis are shown. (B) Liver and ceca of an uninfected control, showing no macroscopic change.

\section{Cloning of parasites}

The cloning was carried out using the limiting dilution method of monoclonal antibodies. The cultured parasites reaching their growth peak were diluted to $\sim 10^{3}$ cells $/ \mathrm{ml}$ with preheated medium, and 1-2 $\mu \mathrm{l}$ of the medium was drawn onto cover slips to observe the parasites under a microscope. Single parasites were labeled and transferred to Eppendorf tubes containing $1 \mathrm{ml}$ of medium and anaerobically cultured in an incubator at $40^{\circ} \mathrm{C}$. After 3-4 days of cultivation, the culture broth containing propagated parasites was inoculated into fresh basal medium at a 1:10 ratio for the propagation of the parasites. The cloned parasite strain was named HM-JSYZ$\mathrm{C}$ and cryopreserved.

\section{Animal inoculation test}

Five 14-day-old SH chickens (purchased from the Institute of Poultry, Chinese Academy of Agricultural Sciences, Jiangsu, China) were inoculated with the HM-JSYZ-C parasite strain through cloacal infection. These chickens were raised in an isolated environment after hatching and fed a drug-free feed formula with free access to water. Animal care and all experiments were performed according to the rules of the Animal 
Experiment Ethics Committee of Yangzhou University. The inoculum used was $10^{5}$ parasites/chicken. Another 5 chickens were inoculated with parasite-free medium as a negative control. After infection, morbidity and clinical symptoms were observed daily, and the chickens were killed after 15 days to observe cecal and liver lesions. The symptoms after inoculation with the HM-JSYZ-C strain in the chickens included depression and diarrhea. Two chickens died within 15 days, and the mortality reached $40 \%$. In the infected group, crater-like circular ulcerative necrotic areas appeared on the liver surface of dead and surviving chickens, the cecal wall was thickened and intestinal emboli formed in the ceca (Fig. 5A). Chickens in the negative control group did not get sick or die, and no significant macroscopic lesions were observed in their livers and ceca (Fig. 5B).

\section{DISCUSSION}

In recent years, the incidence and prevalence of histomoniasis in chicken flocks in China have been on the rise [5]. This may be because, as people's living standards have improved, the demand for organic food has increased. Therefore, locally bred chickens and free-range production systems have become more popular with consumers [6]. In this case, the SH chickens were backyard free ranged. They were free to move outdoors and seek food during the day, making it easy for them to inadvertently consume the eggs of the nematode Heterakis gallinarum - which exist in the external environment and are carriers of histomonads, as well as paratenic hosts, like earthworms, which also exist widely in the environment [8-10]. This is the main reason for this outbreak of histomoniasis. The nematode $H$. gallinarum was not detected from the ceca in this case, which may be related to the use of albendazole (Tongren Pharmaceutical Co. Ltd., Shanghai, China) to expel the parasites in the 45-day-old chickens. In addition, owing to food safety restrictions, the use of drugs for histomoniasis control are prohibited in China (MOA, 2002) [11], which also led to an increase in morbidity caused by histomoniasis.

Histomoniasis can cause a mortality close to $100 \%$ in turkeys, while the mortality in chickens was only $10-20 \%$, which allows chickens to act as reservoir hosts for histomonads $[3,4]$. The isolation and cultivation of $H$. meleagridis in vitro has been reported, however, the main source is from turkey [8] and there were no reports of the strains being isolated from chickens. Histomoniasis is a seasonal disease that occurs most often from April to June. Heterakid eggs can serve as the vectors of $H$. meleagridis, and the coinfection rate is $50.8 \%$ in chickens [8]. The symptoms of histomoniasis in chickens include the bowing of the head and wings, mental fatigue, yellowish green droppings, uplifted peripheral edges and unique crater-like shaped ulcerated lesions in the liver, and swollen ceca. Here, the mortality in naturally infected chickens reached more than $40 \%$, and the mortality in chickens artificially infected with the isolated HM-JSYZ-C strain also reached $40 \%$, which indicated that the virulence of the strain was stronger. The mortality after infection is not only related to the breed and age of the chickens, as well as their feeding style, it is also associated with different genotypes and virulence levels of different geographical isolates of histomonads [12].

Here, the $18 \mathrm{~S}$ rRNA gene was used to identify $H$. meleagridis and diagnose clinical cases. However, it was not the best gene for genotyping histomonads, which may have geographically unique distributions. The ITS and 5.8S rRNA gene are usually used in the C-profiling technique to analyze different genotypes [13]. These are still not perfect markers for genotyping with traditional molecular methods owing to the presence of heterogeneous ITS sequences in a single clone or a mixed infection [14]. Therefore, obtaining more geographical isolates and systematically comparing their genotypes and pathogenicity differences will be the focus of further research.

$H$. meleagridis is too fragile to isolate and culture from infected chickens, and many methods have been tried in our laboratory have been unsuccessful untill recently. We successfully isolated a highly virulent strain of histomonads from the livers of infected chickens for the first time in our laboratory. We used a combination of isolation and culturing, morphological observations and molecular biology techniques to identify that it was true histomonads and was named as HMJSYZ-C strain. These results were more reliable compared with traditional morphological methods. The study of the HMJSYZ-C histomonad strain obtained in this paper will lay a foundation for further investigations into the pathogenicity levels of different geographic histomonad strains in different hosts, and the establishment and evaluation of diagnostic techniques for histomoniasis and attenuated vaccines, as well as the in vivo and in vitro screening of new drugs.

\section{ACKNOWLEDGMENTS}

This work was supported by the National Natural Science 
Foundation of China (grant No. 31772727), the Key Research and Development Plan on Modern Agriculture of Yangzhou City (grant No. YZ2017051), and a project funded by the Priority Academic Program Development of Jiangsu Higher Education Institutions (PAPD). The authors thank Dr. Yanghong Wang and Kuhua Jiao at the Animal Hospital of Yangzhou University for their assistance in collecting suspected cases.

\section{CONFLICT OF INTEREST}

The authors declare that there is no conflict of interest.

\section{REFERENCES}

1. Tyzzer EE. Studies on histomoniasis or "blackhead" infection, in the chicken and turkey. Daedalus 1934; 69: 189-264.

2. Liebhart D, Ganas P, Sulejmanovic T, Hess M. Histomonosis in poultry: previous and current strategies for prevention and therapy. Avian Pathol 2017; 46: 1-18.

3. Dolka B, Żbikowski A, Dolka I, Szeleszczuk P. Histomonosis-an existing problem in chicken flocks in Poland. Vet Res Commun 2015; 39: 189-195.

4. Nguyen DT, Bilic I, Jaskulska B, Hess M, Le DQ, Le Hua LN, Huynh VV, Nguyen ST, Vu-Khac H. Prevalence and genetic characterization of Histomonas meleagridis in chickens in Vietnam. Avian Dis 2015; 59: 309-314.
5. Xu J, Qu C, Tao J. Loop-mediated isothermal amplification assay for detection of Histomonas meleagridis infection in chickens targeting the 18S rRNA sequences. Avian Pathol 2014; 43: 62-67.

6. Wang L, Liu Q, Zheng HE, Wu J, Li X. Development of poultry production clusters in China: A Policy Review. Int J Poult Sci 2014; 13: 292-298.

7. Grabensteiner E, Hess M. PCR for the identification and differentiation of Histomonas meleagridis, Tetrichomonas gallinarum and Blastocystis spp. Vet Parasitol 2006; 142: 223-230.

8. Hauck R, Hafez HM. Experimental infections with the protozoan parasite Histomonas meleagridis: a review. Parasitol Res 2013; 112: 19-34.

9. Hess M, Liebhart D, Bilic I, Ganas P. Histomonas meleagridis--New insights into an old pathogen. Vet Parasitol 2015; 208: 67-76.

10. McDougald LR. Blackhead disease (histomoniasis) in poultry: a critical review. Avian Dis 2005; 49: 462-476.

11. MOA. Chinese ministry of agriculture announcement No. 193 of 5 March 2002 regarding disabled veterinary medicinal products and other compounds list in Food stuffs of Animal Origin. 2002.

12. van der Heijden HM, Landman WJ, Greve S, Peek R. Genotyping of histomonas meleagridis isolates based on internal transcribed spacer-1 sequences. Avian Pathol 2006; 35: 330-334.

13. Hauck R, Balczulat S, Hafez HM. Detection of DNA of Histomonas meleagridis and Tetratrichomonas gallinarum in German poultry flocks between 2004 and 2008. Avian Dis 2010; 54: 1021-1025.

14. Xu J, Qu C, Guo P, Zhuo Z, Liu D, Tao J. Molecular characterization of Histomonas meleagridis in clinical samples of chickens from Eastern China. Thai J Vet Med 2016; 46: 355-361. 